\title{
Evaluating Infrared Thermal Image's Color Palettes in Hot Tropical Area
}

\author{
Romulo L. Olalia Jr., Jenniea A. Olalia, Maynard Gel F. Carse \\ Department of Science and Technology (DOST-PCIEERD), College of Information and Computing Studies, San Carlos College, \\ San Carlos, The Philippines \\ Email: romulo.olalia@sancarloscollege.edu.ph
}

How to cite this paper: Olalia Jr., R.L., Olalia, J.A. and Carse, M.G.F. (2021) Evaluating Infrared Thermal Image's Color Palettes in Hot Tropical Area. Journal of Computer and Communications, 9, 37-49. https://doi.org/10.4236/jcc.2021.911003

Received: October 6, 2021

Accepted: November 8, 2021

Published: November 11, 2021

Copyright $\odot 2021$ by author(s) and Scientific Research Publishing Inc. This work is licensed under the Creative Commons Attribution International License (CC BY 4.0).

http://creativecommons.org/licenses/by/4.0/ (c) (i) Open Access

\begin{abstract}
The use of Infrared Thermal Scanners proved to be very useful in lots of applications. Using different color palettes, temperatures can be well-represented in the resulting image. However, most color palettes in hot tropical places like the Philippines are unsuitable since the ambient temperature is almost the same as the scanned object or person. This study evaluates twelve (12) known and used color palettes in the market to determine the most suitable for tropical places using the edge/border tracing algorithms Sobel-Feldman and Laplacian. The result shows that color palettes with the most colors produce more noise, making it difficult to distinguish the object scanned from the background. On the other hand, color palettes with three or fewer contrasting colors produce crisp and more detailed results. This study helps developers and researchers efficiently use color combinations suitable for hot weather for an effective thermal scanning and image representation.
\end{abstract}

\section{Keywords}

Color Palette, Thermal Infrared Camera, Hot Tropical Area, Edge Border Detection

\section{Introduction}

On March 11, 2020, World Health Organization (WHO) declared COVID-19 a pandemic, citing over 118,000 cases of coronavirus infections in over 100+ countries and places around the world [1]. This means that public health emergency becomes an international concern that makes almost all nation device guidelines, adapt protocols, close borders [2] [3] and boundaries [4], and even making market and supply chain disruption [5].

In these days of uncertainty where the effect of the COVID-19 has been af- 
fecting all aspects of human lives, safety measures and protocols are necessary to ensure the safety of the public and the frontliners themselves. The Administrative Order No. 2020-0016 issued by the Department of Health, also known as "Minimum Health System Capacity Standards for COVID-19 Preparedness and Response Strategies," suggests physical distancing of at least 1 meter apart at all times [6]. The Inter-Agency Task Force (IATF) adopted these minimum health standards for the Management of Emerging Infectious Disease in almost all its resolutions. Section A-4 of the IATF Resolution No. 13 (2020) states that the Philippine National Police (PNP) was instructed to conduct inspection procedures in checkpoints to observe protocols. This includes inspection of necessary documents and using an infrared thermal scanner to filter possible COVID-19 positive individuals. The same practice has been adopted by the private and government offices and institutions, which was mandated through the guidelines issued by the IATF [7].

IRT's (also known as IR thermal cameras) and Non-Contact IR Thermometer are the only currently viable temperature measurement approaches for mass monitoring infectious diseases such as COVID-19. NCITs are the most popular fever screening, and all IRT's are approved by US Food and Drug Administration [8]. Benefits of using thermal imaging systems include effective mass fever screening, faster than forehead thermal scanner, and accurate skin temperature reading [9]. Although issues on its effectiveness are undeniable [10], IT thermal cameras are still applauded as a quick detector of human temperature. IR Thermal cameras are not perfect, but they can surely help control the spread of the virus [11].

As recommended by the US-FDA in their general guidelines for the preparation of Thermal Imaging Systems (Infrared Thermographic Systems/Thermal Imaging Cameras), the room temperature should be around $20^{\circ} \mathrm{C}-24^{\circ} \mathrm{C}$ and relative humidity of $10 \%-50 \%$ [9]. Almost the same configuration was recommended by a study done by Ghassemi et al. According to their research, the room temperature should be maintained at $18^{\circ} \mathrm{C}$ to $24^{\circ} \mathrm{C}$ and relative humidity between $10 \%$ and $75 \%$ [8]. This suggestion is ideal for humid places, and the ambient temperature is cold. However, in hot tropical areas like in the Philippines, the use of IR thermal poses a challenge [12].

The Philippines' climate is usually tropical and maritime, which means it is characterized by high temperature and humidity, and abundant rainfall [13]. The mean annual temperature of the Philippines is $26.60^{\circ} \mathrm{C}$. January is the coolest month at $25.50^{\circ} \mathrm{C}$ during January and the warmest month is in May, with an average temperature of $28.3^{\circ} \mathrm{C}$ and humidity of $60 \%$. On May 10, 2021, Dagupan City, Pangasinan, reaches a record high heat index or temperature of $51^{\circ} \mathrm{C}$, which could cause health problems such as heat cramps and exhaustion, and heat stroke [14]. Two days following the record high heat index, on May 12, 2021, Dagupan City again reached a heat index of $52^{\circ} \mathrm{C}$ at $2 \mathrm{pm}$ [15]. This was followed by another record-high heat index of $53^{\circ} \mathrm{C}$ at the same time and loca- 
tion [16].

The unstableness of temperature in the Philippines poses a significant problem in thermal imaging. The humid subtropical climates in some regions are considered problematic with current technologies [12]. As the air temperature rises, the humidity of the surroundings decreases, making the heat index spike. The same result was observed by Tran et al. [17) in their study about ambient temperature and relative humidity effects. According to their research, variation in the relative humidity occurred because the air pressure determines the saturation of vapor pressure. This contrasts with the recommendation of the US Food and Drugs Administration in the operation of IR thermal cameras. According to US-FDA, an infrared thermal imaging device should be suitable and set up and operated correctly [9].

This study aims at evaluating currently known color palettes for thermal imaging. The evaluation result is the determination of the most appropriate color palettes that will be suitable for tropical and humid regions like the Philippines. The result of the study helps developers and researchers efficiently use color combinations ideal for hot weather for an effective thermal scanning and image representation.

\section{Literature Review}

\subsection{Problem in Thermal Imaging in Hot Tropical Area}

If the ambient temperature of the surroundings reaches the temperature range of human subjects, rendering the thermal image can be a problem since color differentiation can be more challenging. As stated by the National Library of Medicine of the United States, the average normal body temperature is between $36.1^{\circ} \mathrm{C}$ to $37.20^{\circ} \mathrm{C}$, based on some studies. A temperature above $38^{\circ} \mathrm{C}$ most often means the person has a fever caused by an illness or infection [18]. For practical clinical purposes, a person is considered febrile (having or showing fever symptoms) when the oral temperature exceeds $37.5^{\circ} \mathrm{C}$ [19]. If the temperature of the surrounding area and the human body are almost the same or overlap with each other, this will cause a lot of noise in the image being rendered using color palettes that are available with IR thermal scanners in the market. This is the main problem of this study. This study aims to help developers and other researchers determine the best color palettes for such peculiar conditions of temperature.

\subsection{Image Capture and Lighting}

Capturing thermal images can be in itself a challenge. Research suggests the use of an aerial thermal scanner and artificial-intelligence-enabled Internet-of-Things to safely capture human temperature over a safe distance [20]. Capturing thermal images using UAVs (unmanned aerial vehicles) is also gaining interest [21]. IoT-enabled applications and devices were also used in different studies. This IoT integration includes assessing thermal comforts for people [22] and thermal comforts for building design [23]. In this study, IoT modules were integrated to 
comprise the thermography setup. These IoT modules help capture thermal images of subjects in an easy-to-setup manner for further edge detection processing.

While some researches on thermography use light to mimic a feeling of warmth [24], subject to the evaluator's perception of virtual reality, light has no direct correlation with thermal scanning [25]. Other researches also suggest that the use of color affects or influence temperature perception, also known as "hueheat perception" [26]. Even using light-emitting diode (LED) light directly with an adjustable correlated thermal color temperature fails to support the correlation of light with skin temperature [27]. Additionally, sunlight does not affect human temperature. The research results show that people who have short exposure (around 30 minutes) to daylight during daytime affect the subject's thermal perception. Still, the subject's skin temperature was not affected by daylight in four different testing rooms [28]. In this research, although the light is present on the thermography setup, the researchers do not consider the effect of the light on the skin temperature being scanned by the infrared thermal scanner.

\subsection{Color Palettes for Thermal Imaging}

Color palettes are a very important aspect of representing temperature values. Human ability to see clearly in dark or poor visibility areas is very restrained but in thermal imaging, lighting is not a big problem. With the proper color combination, the rendering of thermal images can be very effective and appealing. In a medical sense, color palette selection helps in the detection or recognition process [29]. With color palettes, facial thermal images can be captured and analyzed using mathematical algorithms to extract meaningful features [30].

Choosing a suitable color palette is the key to effective thermal imaging. Switching from different palettes changes the appearance of a scene and highlights critical areas of an image without altering the scanned temperature data. Teledyne FLIR, an imaging company that designs, develops, and manufactures thermal imaging technologies, uses its standard color palettes designed for various environments, purposes, and conditions. Among those palettes include WhiteHot, Sepia, Rainbow, Black-Hot, Outdoor, Artic, Iron Gradient [31], Lava, Night Vision, BGY, Electro, and Candy.

However, these color palettes are not optimized in seasonally hot tropical regions like the Philippines. Implementing and setting an IR thermal device/system during this season poses a challenge. This study analyzes each available color palette in the market. In evaluating each color palette, Laplacian and Sobel-Feldman edge detection algorithms will be used.

\section{Methodology}

There are 12 color palettes used in this study. Using a color-gradience tool (Figure 1), each known color palette used in thermal imaging was represented. The application is capable of accepting six colors and fills the gradient between 


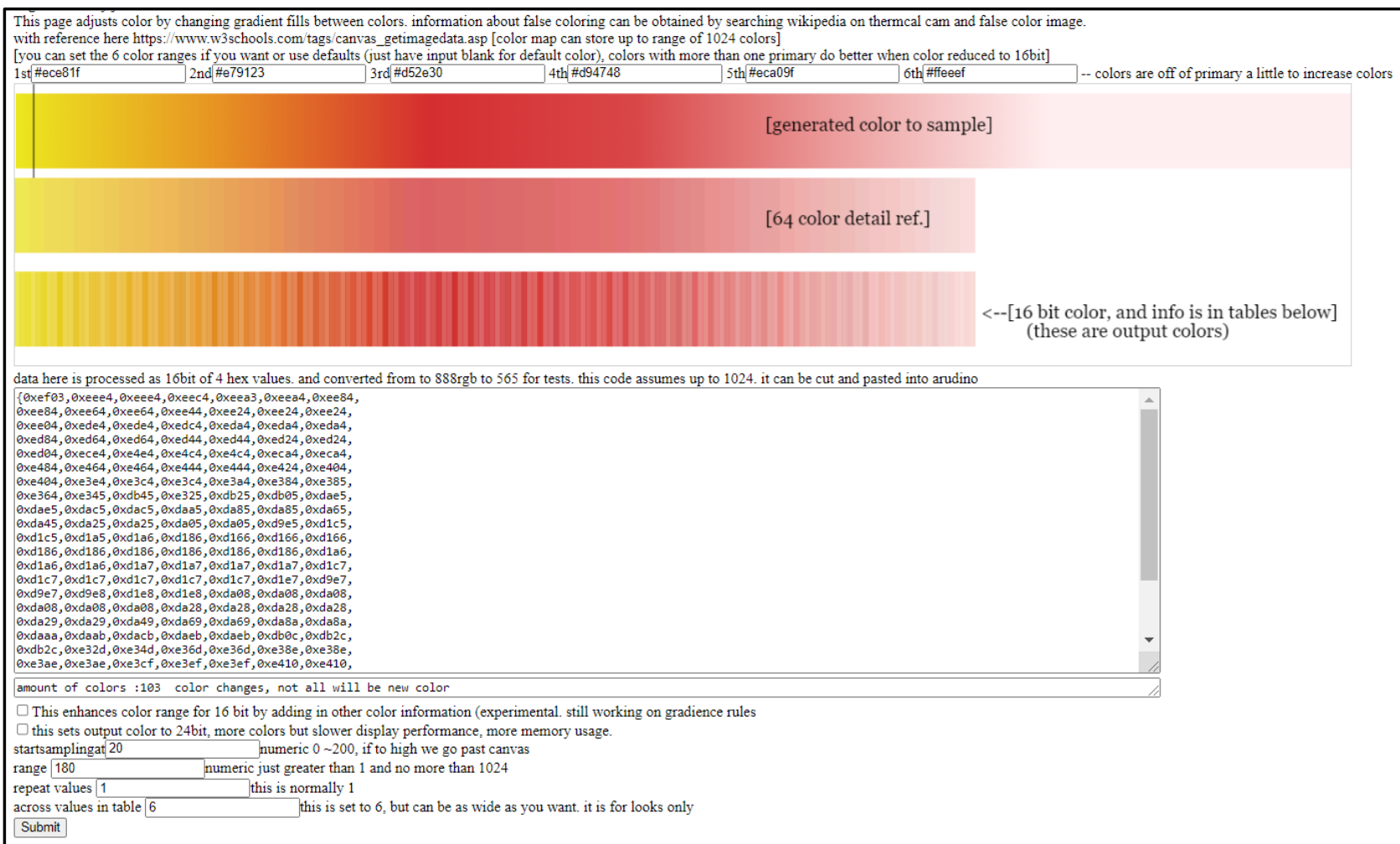

Figure 1. The color gradience generation tool.

each color. The color palette is divided into six measures. The color code generated is a 16-bit color of 4 hexadecimal values. These values are converted from 888 RGB to 565 hexadecimal for testing. These values are pasted in a code for rendering.

All of the color palettes are subjected to an edge/border tracing algorithm. This is a mathematical method of determining how the color palettes display the degree of detailedness of the object being scanned. This is very significant because if the surrounding ambient temperature is almost the same as the human being observed, the noise will be visible in the background. Figure 2 shows a sample output using Laplacian and Sobel-Feldman algorithm.

The Laplacian method is an edge/border detection algorithm that can determine if a rendered thermal image can be scientifically outlined. With such, subjective evaluation can be done on the thermal image, using different color palettes, if subjects, i.e., human, etc., can be recognized from the background. The equation was first discovered and introduced by a French mathematician and astronomer, Pierre-Simon Laplace. Laplace's equation states that the sum of the second-order partial derivatives of $\mathrm{R}$, the unknown function, with respect to the Cartesian coordinates, equals zero:

$$
\frac{\partial^{2} R}{\partial x^{2}}+\frac{\partial^{2} R}{\partial y^{2}}+\frac{\partial^{2} R}{\partial z^{2}}=0
$$

The sum on the left is commonly represented by the expression $\nabla^{2} R$, in which the symbol $\nabla^{2}$ is called the Laplacian, or the Laplace operator. 


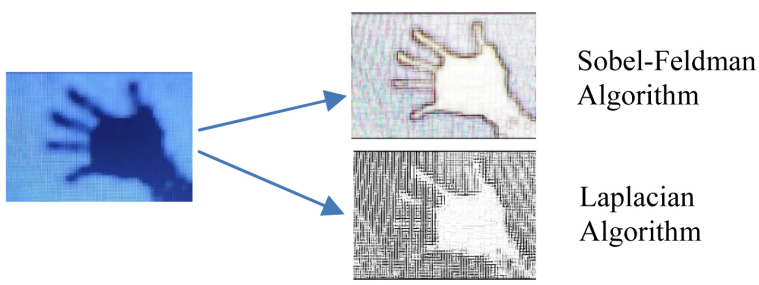

Figure 2. Sample result of edge/border tracing using Laplace and Sobel-Feldman algorithms.

On the other hand, the Sobel-Fieldman operator is also an edge detection algorithm that emphasizes the edges of an image. This computer vision algorithm is used in image processing to extract the object's contour. This works by using two $3 \times 3$ kernels for ins convolution:

$$
\begin{array}{|l|l|l|}
\hline-1 & 0 & +1 \\
\hline-2 & 0 & +2 \\
\hline-1 & 0 & +1 \\
\hline
\end{array}
$$

$\mathrm{Gx}$

$$
\begin{array}{|c|c|c|}
\hline+1 & +2 & +1 \\
\hline 0 & 0 & 0 \\
\hline-1 & -2 & -1 \\
\hline \multicolumn{3}{|c|}{\text { Gy }}
\end{array}
$$

These kernels $\left(G_{x}\right.$ and $\left.G_{y}\right)$ can produce a separate measurement of the gradient component for each orientation. These can be combined to find the absolute magnitude of the gradient at each point and the orientation of that gradient using

$$
G=\sqrt{G_{x}^{2}+G_{y}^{2}}
$$

Using this information, gradients direction can also be calculated using

$$
\theta=\operatorname{atan} 2\left(G_{y}, G_{x}\right)
$$

To capture thermal images, the researchers created a thermal scanning setup (Figure 3) that includes an ESP32 electronic board, MLX90640 IR thermal camera, and a 1.44" LCD IPS screen display. During benchmark, the LCD screen display is capable of filling the entire screen in 42.33 milliseconds. This gives the LCD roughly around 24 frames per second.

The setup for the test includes the thermal scanner atop a table, as sown in Figure 4. The experiment was set up in a normal room with fluorescent lighting on top of the thermal scanner at a distance of 76 inches. There is no external lighting in the setup. The object being scanned is 32 inches away from the thermal scanner. Although the temperature of the fluorescent light is higher than the room temperature, it doesn't affect the temperature of the object being scanned. Moreover, the intensity of the light does not affect the operation of the thermal scanner since the thermal scanner can work even in the absence of light.

Although humidity can affect the heat index, humidity is not a factor to be considered in this study. If humidity is to be factored in with the temperature, the heat index can be computed. The heat index, also known as apparent temperature, is what temperature feels like to the human body after relative humidity 


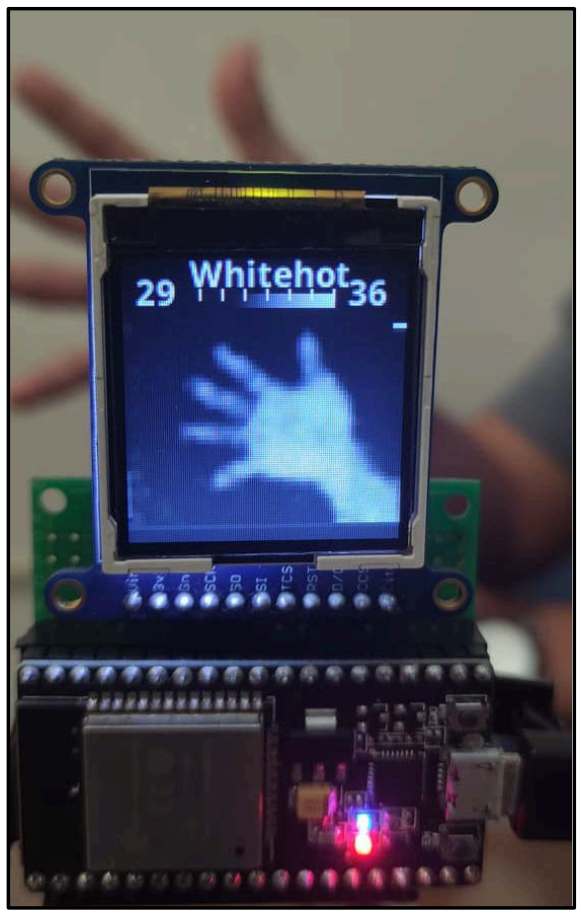

Figure 3. Thermal imaging setup.

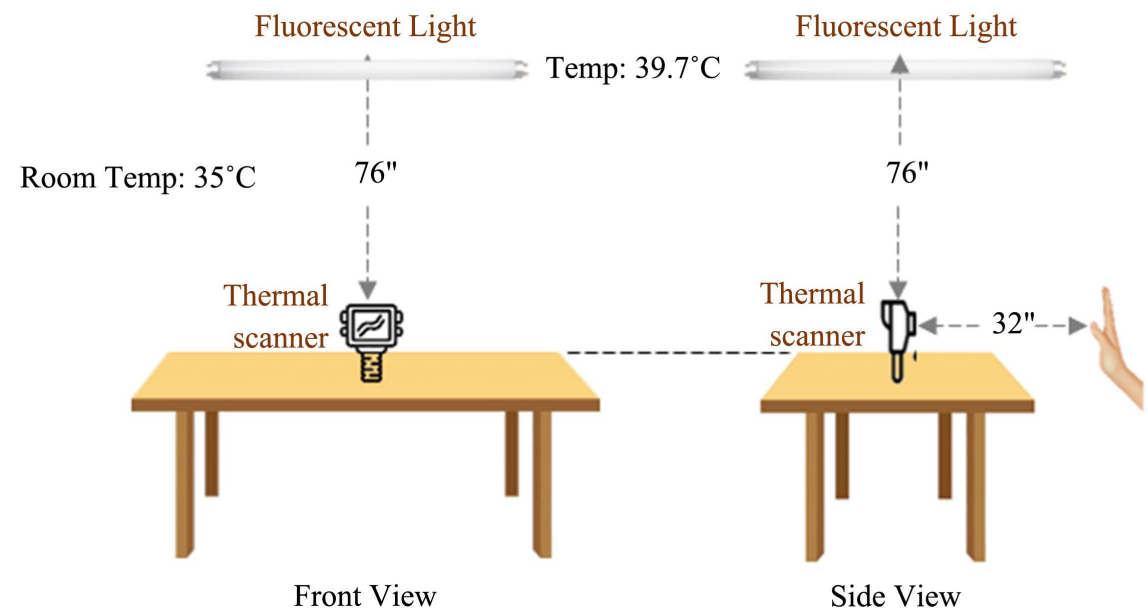

Figure 4. Thermography setup.

is mixed with the air temperature [32]. The temperature being captured by a thermal camera is the actual temperature of the body or an object and not the feel of the temperature.

\section{Results and Discussion}

Using the color-gradience tool, six key colors, in hex, were used for the gradient generation. From 1 color to another, gradient colors were generated, as shown in Figure 5.

These color gradients were converted into hex equivalent, which was used to generate the color palette based on the values given by the thermal scanner. The 


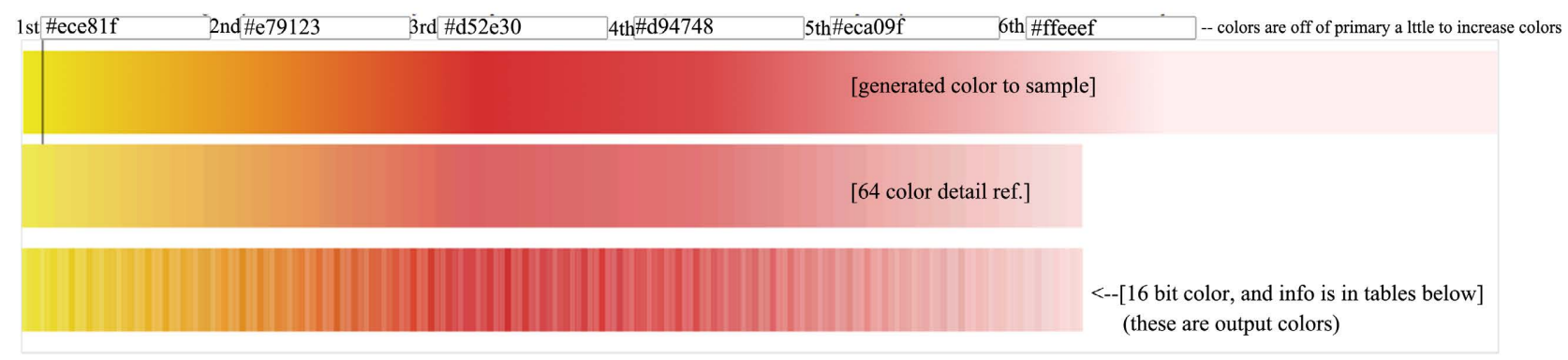

Figure 5. Generated gradient colors from 6 key colors.

code below represents the 103 colors being generated by the application from the six key colors.

$\{0$ xef 03,0 xeee 4,0 xeee 4,0 xeec 4,0 xeea 3,0 xeea 4,0 xee 84,0 xee 84,0 xee $64,0 x$ ee 64,0 xee 44,0 xee 24,0 xee 24,0 xee 24,0 xee 04,0 xede 4,0 xede 4,0 xedc 4,0 xeda 4,0 xeda 4,0 xeda 4,0 xed 84,0 xed 64,0 xed 64,0 xed 44,0 xed 44,0 xed 24,0 xed 24,0 xed0 4,0 xece 4,0 xe 4 e 4,0 xe 4 c 4,0 xe 4 c 4,0 xeca 4,0 xeca 4,0 xe 484,0 xe 464,0 xe 4 64,0 xe 444,0 xe 444,0 xe 424,0 xe 404,0 xe 404,0 xe 3 e 4,0 xe $3 c 4,0 x e 3 c 4,0 x e 3 a 4$, 0 xe 384,0 xe 385,0 xe 364,0 xe $345,0 x d b 45,0 x e 325,0 x d b 25,0 x d b 05,0 x d a e 5,0 x d$ ae $5,0 x d a c 5,0 x d a c 5,0 x d a a 5,0 x d a 85,0 x d a 85,0 x d a 65,0 x d a 45,0 x d a 25,0 x d a 25$ , 0xda05, 0xda05, 0xd9e5, 0xd1c5, 0xdlc5, 0xdla5, 0xdla6, 0xd186,0xd166, 0x d166, 0xd166, 0xd186, 0xd186,0xd186, 0xd186, 0xd186, 0xd186, 0xd1a6, 0xd1a 6,0xd1a6,0xd1a7, 0xd1a7, 0xd1a7, 0xd1a 7, 0xd1c7, 0xd1c7, 0xd1c7, 0xd1c7, 0 xd1c7, 0xd1c7,0xdle7,0xd9e7,0xd9e7,0xd9e8,0xd1e8,0xd1e8,0xda08,0xda $08,0 x d a 08,0 x d a 08,0 x d a 08,0 x d a 08,0 x d a 28,0 x d a 28,0 x d a 28,0 x d a 28,0 x d a 29$, 0xda29, 0xda49,0xda69,0xda69, 0xda8a, 0xda8a, 0xdaaa, 0xdaab, 0xdacb, 0xd aeb, 0 xdaeb, 0 xdb $0 \mathrm{c}, 0 \mathrm{xdb} 2 \mathrm{c}, 0 \mathrm{xdb} 2 \mathrm{c}, 0$ xe $32 \mathrm{~d}, 0 \mathrm{xe} 34 \mathrm{~d}, 0 \mathrm{xe} 36 \mathrm{~d}, 0 \mathrm{xe} 36 \mathrm{~d}, 0 \mathrm{xe} 38 \mathrm{e}$ , 0xe38e, 0xe3ae, 0xe3ae, 0xe3cf, 0xe3ef, 0xe3ef, 0xe410, 0xe410, 0xe430, 0x e 430,0 xec 51,0 xec 71,0 xec 71,0 xec 92,0 xec 92,0 xecb 2,0 xecd 2,0 xecd 3,0 xec $f$ 3,0 xec 13,0 xed13,0xed14,0xed 34,0 xed 34,0 xed55, 0xed55, 0xed75, 0xed75, 0 xf596,0xf596,0xf5b6,0xf5b6,0xf5d7,0xf5d7,0xf5f7,0xf5f7,0xf618,0xf 6 $38,0 x f 638,0 x f 638,0 x f 659,0 x f 659,0 x f 679,0 x f e 9 a, 0 x f e 9 a, 0 x f e 9 a, 0 x f e b a$, Oxfeba\};

Applying this color scale for a given color palette, thermal values from the thermal scanner can be represented. These steps were done to represent all 12 color palettes. Figure 6 shows the representation of thermal values in different color palettes.

Images rendered using color palettes were captured by a smartphone then compiled for subjection to the edge detection algorithms. The edge or border detection algorithms were used to determine the clarity or detailedness of the scanned image while minimizing the noise around it. The evaluation focuses on these two factors to select the most appropriate. Figure 7 shows all 12 palettes after subjection to the Sobel-Feldman algorithm.

After subjecting all color palettes to the Sobel-Feldman algorithm, the result shows that the White-Hot color palette displays less noise in the background and detailed pattern of the scanned hand compared to others. Moreover, layered border-color means varying the detailed temperature of the object being scanned, which is significant to thermal imaging. On the opposite, the Black-Hot color palette shows a clean object while having a consistent minimal noise in the background. Almost similar to Black-Hot is the Outdoor color palette with an additional color rendition for a higher temperature inside the object. 


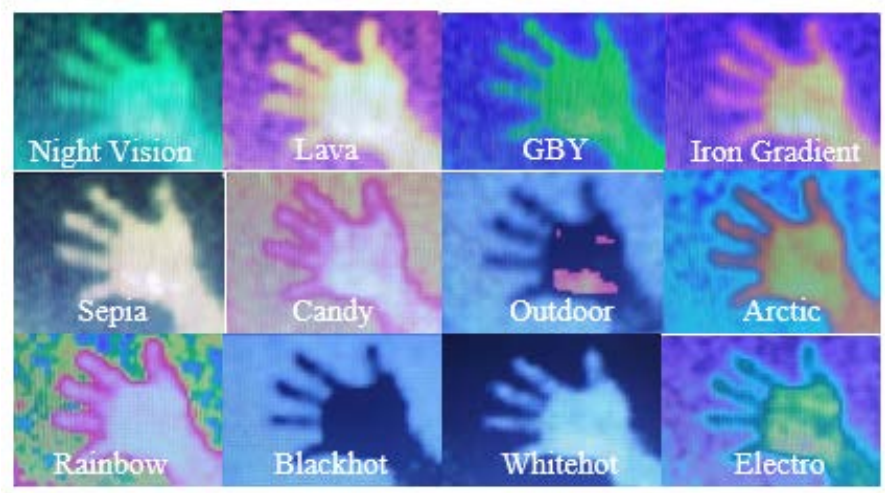

Figure 6. Color representation of known thermal palettes.

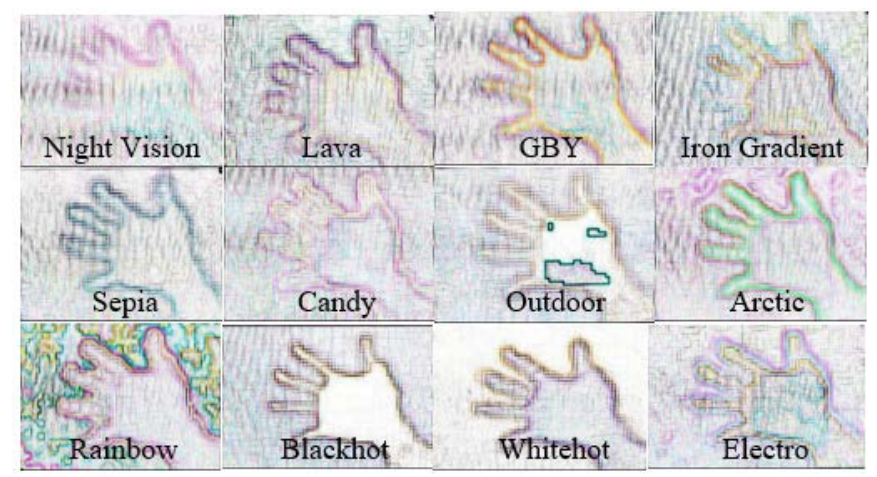

Figure 7. Sobel-Feldman edge detection of each color palettes.

The Sepia palette has a clear border, although the background noise can be observed extending in the inner part of the object. Color palettes such as Night Vision and Iron Gradient have distorted or partial completeness, missing 1 to 2 fingers. Most of the color palettes have a noisy background that almost blended with the object. This makes it difficult to differentiate the temperature of the environment and the object being thermally scanned. Although the object is still recognizable, noises are very dominant in the background and the foreground. This observation is most noticeable in the rainbow palette.

With the use of the Laplacian edge tracing algorithm, the same thermal images are subjected. Figure 8 shows the result of the tracing. Consistently, BlackHot color palettes display an excellent render of the object scanned, showing a clear hand with a consistent background. Additionally, the White-Hot color palette shows solid foreground but a bit distorted background. The Outdoor color palette, which is a color higher compared to Black-Hot, renders almost the same, with additional details on the hotter part of the object. Lava, Iron Gradient, Artic, and Electro shows a bit of a border but are barely visible. Color palettes that comprise more colors blend the foreground and background, and almost no visible trace can be seen.

Black-Hot and White-Hot color palettes both render consistent results on Sobel Feldman and Laplacian edge/border tracing. This consistency of results suggests that both are suitable for tropical weather like the Philippines. Outdoor 
color palettes, which are almost the same as Black-Hot, offer additional details on temperature but are less traced.

To further clear the image of unwanted noise, gradient magnitude thresholding or lower bound cut-off suppression was done. The high threshold value is set at 0.20 and the low threshold value is set to 0.10 .

The presence of noise or incompleteness of the image scanned can be correlated with the number of primary colors used during the generation of key colors. Figure 9 shows that palettes with a minimum number of colors have clearer rendering compared to palettes with more colors.

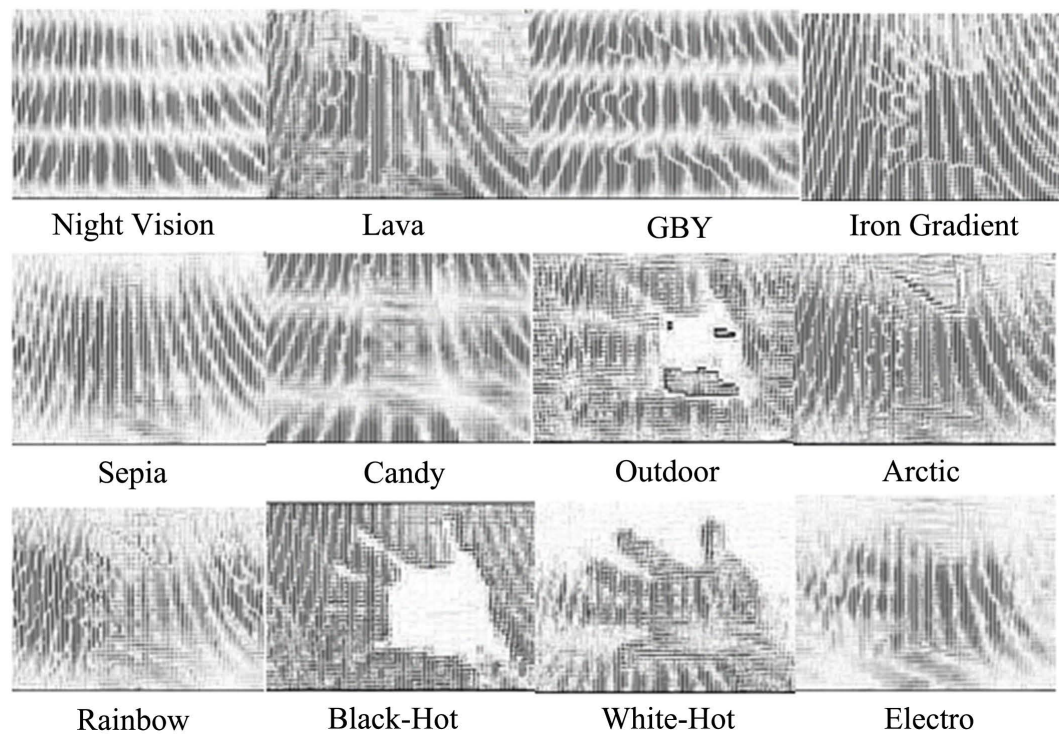

Figure 8. Laplacian edge detection of each color palette.

\begin{tabular}{|c|c|c|}
\hline Detected Edges & $\begin{array}{c}\text { Color } \\
\text { Palettes }\end{array}$ & $\begin{array}{c}\text { No. of } \\
\text { Primary } \\
\text { Colors Used }\end{array}$ \\
\hline & Rainbow & 6 \\
\hline & Electro & 6 \\
\hline & Arctic & 6 \\
\hline
\end{tabular}

\begin{tabular}{|c|c|c|}
\hline Detected Edges & $\begin{array}{c}\text { Color } \\
\text { Palettes }\end{array}$ & $\begin{array}{c}\text { No. of } \\
\text { Primary } \\
\text { Colors Used }\end{array}$ \\
\hline & BGY & 5 \\
\hline & Sepia & 4 \\
\hline & Outdoor & 4 \\
\hline & Night Vision & 4 \\
\hline
\end{tabular}

Figure 9. Laplacian edge detection of each color palette. 


\section{Conclusions and Recommendation}

During the experiment, color palettes were utilized successfully by determining key colors then generating the gradients between them. These gradient colors can be used to represent small details of temperature based on the scanned thermal values of the thermal scanner. With the rendering of thermal values into color representation, thermal images based on palettes can be used for edge/border detection to assess the detailedness of the object scanned scientifically.

Based on the observation with color palettes and their rendering on the Sobel-Feldman algorithm, it is concluded that the more color the palette has, the more noise it generates. Although color shows more borders, the object being scanned is challenging to distinguish. The use of minimal contrasting colors, however, renders a solid, detailed image that is more recognizable.

Using the Laplacian algorithm, the result further supports the findings with the Sobel-Feldman algorithm. While most colored palettes render noisy backgrounds and incomplete images, two-colored palettes, such as the black-hot and white-hot, still render a more solid and detailed image.

The use of minimal contrasting colors to distinguish the object being scanned from the background is therefore recommended to be used in hot tropical areas like the Philippines. The result will greatly help developers of thermal scanners to effectively use appropriate color palettes in their prototypes or products to be used not only in the Philippines but also in other tropical places.

Furthermore, it is recommended that future researchers should improve the capturing of the image to minimize the noise in the background. They should confirm if the noise in the captured image is brought about by the low refresh or frame rate of the screen display.

\section{Acknowledgements}

The researchers acknowledge and give their sincerest gratitude to the Philippine Council for Industry, Energy, and Emerging Technology Research and Development, Department of Science and Technology (DOST-PCIEERD) for funding this project. Moreover, the researchers bestow their earnest appreciation to the support of the College of Information and Computing Studies, San Carlos College, Philippines. To God be the Glory!

\section{Conflicts of Interest}

The authors declare no conflicts of interest regarding the publication of this paper.

\section{References}

[1] Ducharme, J. (2020) World Health Organization Declares COVID-19 a "Pandemic." Here's What That Means. Time Magazine. https://time.com/5791661/who-coronavirus-pandemic-declaration

[2] IATF (2020) IATF Resolution No. 9 Series of 2020. Recommendation for the Man- 
agement of the Coronavirus Disease 2019 (COVID-19) Situation. https://doh.gov.ph/sites/default/files/health-update/IATF-RESO-11.pdf

[3] Reichert, C. (2020) The European Union Is Closing Its Borders in COVID-19 Measures. C/Net.

https://www.cnet.com/news/the-european-union-is-closing-its-borders-in-covid-19 -measures

[4] CGTN (2020) COVID-19 Global Roundup: World Closes Borders, Restricts Travel to Contain Coronavirus Spread. https://news.cgtn.com/news/2020-03-15/COVID-19-Global-Roundup-World-closes -borders-restricts-travel-OS9E0r1wOY/index.html

[5] Bachman, D. (2020) The Economic Impact of COVID-19 (Novel Coronavirus) Deloitte Insights.

https://www2.deloitte.com/us/en/insights/economy/covid-19/economic-impact-cov id-19.html

[6] DOH (2020) Administrative Order No. 2020-0016-Minimum Health Capacity Standard for COVID-19 Preparedness and Response Strategies.

[7] IATF (2020) IATF Resolution No. 38, Series of 2020.

[8] Ghassemi, P., et al. (2018) Best Practices for Standardized Performance Testing of Infrared Thermographs Intended for Fever Screening. PLOS ONE, 13, e0203302. https://doi.org/10.1371/journal.pone.0203302

[9] US-FDA (2021) Thermal Imaging Systems (Infrared Thermographic Systems/Thermal Imaging Cameras). US Food \& Drug Administration.

https://www.fda.gov/medical-devices/general-hospital-devices-and-supplies/therma l-imaging-systems-infrared-thermographic-systems-thermal-imaging-cameras

[10] Harwell, D. (2020) Thermal Scanners Are the Latest Technology Being Deployed to Detect the Coronavirus. But They Don't Really Work. The Washington Post. https://www.washingtonpost.com/technology/2020/05/11/thermal-scanners-are-late st-technology-being-deployed-detect-coronavirus-they-dont-really-work

[11] Ketchell, M. (2020) Thermal Cameras Aren't Perfect, But They Can Help Control the Coronavirus Pandemic. The Conversation.

https://theconversation.com/thermal-cameras-arent-perfect-but-they-can-help-cont rol-the-coronavirus-pandemic-141701

[12] Thomson, S.J., et al. (2012) Potential and Challenges in Use of Thermal Imaging for Humid Region Irrigation System Management. Journal of Agricultural Science, 4, 103. https://doi.org/10.5539/jas.v4n4p103

[13] DOST-PAGASA (n.d.) Climate in the Philippines. http://bagong.pagasa.dost.gov.ph/information/climate-philippines

[14] Flores, H. (2021) Philippines Records 51 Degrees Celsius Heat Index in Pangasinan. PhilStar Global.

https://www.philstar.com/nation/2021/05/10/2097066/philippines-records-51-degre es-celsius-heat-index-pangasinan

[15] Rappler (2021) Dagupan City Heat Index Hits $52{ }^{\circ} \mathrm{C}$ on May 12. Rappler. https://www.rappler.com/nation/weather/dagupan-city-pangasinan-heat-index-ma $\mathrm{y}-12-2021$

[16] Rappler (2021) Dagupan City Sets New 2021 High with $53{ }^{\circ} \mathrm{C}$ Heat Index on May 14. Rappler.

https://www.rappler.com/nation/weather/dagupan-city-pangasinan-heat-index-ma y-14-2021

[17] Tran, Q.H., et al. (2017) Effects of Ambient Temperature and Relative Humidity on 
Subsurface Defect Detection in Concrete Structures by Active Thermal Imaging. Sensors (Switzerland), 17, 1-18. https://doi.org/10.3390/s17081718

[18] Vorvick, L.J. (2021) Body Temperature Norms. U.S. National Library of Medicine. https://medlineplus.gov/ency/article/001982.htm

[19] Del Bene, V.E. (1990) Clinical Methods: The History, Physical, and Laboratory Examinations. 3rd Edition, National Center for Biotechnology Information, Bethesda.

[20] Barnawi, A., et al. (2021) Artificial Intelligence-Enabled Internet of Things-Based System for COVID-19 Screening Using Aerial Thermal Imaging. Future Generation Computer Systems, 124, 119-132. https://doi.org/10.1016/j.future.2021.05.019

[21] Pardo, J.A., et al. (2017) Wireless Communication System for the Transmission of Thermal Images from a UAV. 2017 CHILEAN Conference on Electrical, Electronics Engineering, Information and Communication Technologies (CHILECON), Pucon, 18-20 October 2017, 1-5. https://doi.org/10.1109/CHILECON.2017.8229690

[22] Brik, B., et al. (2021) An IoT-Based Deep Learning Approach to Analyse Indoor Thermal Comfort of Disabled People. Building and Environment, 203, Article ID: 108056. https://doi.org/10.1016/j.buildenv.2021.108056

[23] Shahinmoghadam, M., et al. (2021) BIM- and IoT-Based Virtual Reality Tool for Real-Time Thermal Comfort Assessment in Building Enclosures. Building and Environment, 199, Article ID: 107905. https://doi.org/10.1016/j.buildenv.2021.107905

[24] Chinazzo, G., et al. (2017) The Effect of Short Exposure to Coloured Light on Thermal Perception: A Study Using Virtual Reality. Proceedings of the Lux Europa 2017, Lubljana, 18-20 September 2017, 273-279. https://infoscience.epfl.ch/record/231194

[25] Li, B., et al. (2013) Datacenter Scale Evaluation of the Impact of Temperature on Hard Disk Drive Failures. Proceedings of the 14th International Conference on Artificial Intelligence and Statistics, Vol. 15, 434-442.

[26] Ziat, M., et al. (2016) A Century Later, the Hue-Heat Hypothesis: Does Color Truly Affect Temperature Perception? In: Bello, F., Kajimoto, H. and Visell, Y., Eds., Haptics. Perception, Devices, Control, and Applications, Springer International Publishing, Berlin, 273-280. https://doi.org/10.1007/978-3-319-42321-0 25

[27] Baniya, R.R., et al. (2018) The Effect of Correlated Colour Temperature of Lighting on Thermal Sensation and Thermal Comfort in a Simulated Indoor Workplace. Indoor and Built Environment, 27, 308-316. https://doi.org/10.1177/1420326X16673214

[28] Chinazzo, G., et al. (2019) Daylight Affects Human Thermal Perception. Scientific Reports, 9, 1-15. https://doi.org/10.1038/s41598-019-48963-y

[29] Agrawal, D. and Karar, V. (2018) Color Palette Selection in Thermal Imaging for Enhancing Situation Awareness during Detection-Recognition Tasks. 2018 International Conference on Recent Innovations in Electrical, Electronics Communication Engineering (ICRIEECE), Bhubaneswar, 27-28 July 2018, 1227-1232. https://doi.org/10.1109/ICRIEECE44171.2018.9008486

[30] Wang, Z.-H., et al. (2020) A Novel Facial Thermal Feature Extraction Method for Non-Contact Healthcare System. IEEE Access, 8, 86545-86553.

https://doi.org/10.1109/ACCESS.2020.2992908

[31] Flir, T. (2021) Picking a Thermal Color Palette. https://www.flir.com/discover/industrial/picking-a-thermal-color-palette

[32] NWS (n.d.) What Is the Heat Index? National Weather Services. https://www.weather.gov/ama/heatindex 